Bradley, Andrew; Gerard, France; Barbier, Nicolas; Zelazowski, Przemyslaw; Weedon, Graham; Huntingford, Chris; Anderson, Liana; Eduardo, Luiz. 2008 Exploring the biophysical drivers of Amazon phenology: preparing data sets to improve dynamic global vegetation models. In: 2008 IEEE International Geoscience \& Remote Sensing Symposium, Boston, 6-11 July 2008. Institute for Electrical and Electronics Engineers.

This version available at http://nora.nerc.ac.uk/5512/

NERC has developed NORA to enable users to access research outputs wholly or partially funded by NERC. Copyright and other rights for material on this site are retained by the authors and/or other rights owners. Users should read the terms and conditions of use of this material at http://nora.nerc.ac.uk/policies.html\#access 


\title{
EXPLORING THE BIOPHYSICAL DRIVERS OF AMAZON PHENOLOGY: PREPARING DATA SETS TO IMPROVE DYNAMIC GLOBAL VEGETATION MODELS.
}

\author{
Andrew Bradley ${ }^{1}$, France Gerard ${ }^{2}$, Nicolas Barbier ${ }^{3}$, Przemyslaw Zelazowski ${ }^{3}$, Graham Weedon ${ }^{2}$, Chris Huntingford ${ }^{2}$, Liana Anderson ${ }^{3}$, \\ and Luiz Eduardo O.C. de Aragão ${ }^{3}$
}

\author{
${ }^{1}$ Centre for Ecology and Hydrology, Monks Wood, Abbots Ripton, Huntingdon, Cambridgeshire, PE28 2LS, United Kingdom. \\ ${ }^{2}$ Centre for Ecology and Hydrology, Wallingford, Oxfordshire, OX10 8BB, United Kingdom. \\ ${ }^{3}$ Environmental Change Institute, Oxford University Centre for the Environment, Oxford, OX1 3QY, United Kingdom.
}

*Contact information: E: abrad@ceh.ac.uk: T: +0044 (0) 1487 772400: F: +0044 (0) 1487773467

\begin{abstract}
We explore the relative influence of biophysical drivers on phenology to assist validation and parameterization of Dynamic Global Vegetation Models. Using 6.8 years of MODIS data we created a vegetation index time series to map the spatial variability of vegetation phenology in the Amazon. TRMM and CERES data were used as a measure of two biophysical variables, precipitation and net radiation respectively. Using a Fourier transform and cross spectral analysis two aspects were considered from these data, the coincidence of: (A) spatial patterns, presence and strength in the annual cycle, and (B) the coherency and phase differences between the phenology and the biophysical variables. Using the Amazon as a study area we find that the coincidence between phenology and the drivers in annual power strength was not linear and in an area of high coherency we found radiation and phenology was almost in phase, whilst precipitation was not. The correspondence of slightly subdued annual phenology with strong annual radiation indicated that other drivers also influence the strength of the phenology.
\end{abstract}

Index Terms - Satellite applications, Vegetation mapping, Fourier transform, spectral analysis, modeling.

\section{INTRODUCTION}

Global climate models are a major tool used to simulate the future course of climate and influence policy to mitigate anthropogenic influence on the earth system [1]. However there is still a degree of uncertainty in these models in both the magnitude of climate change and the impacts of climate change on the sustainability of ecosystems. For example by 2050 several model scenarios predict major drying in the Amazon causing a dieback of Amazon vegetation [2] [3], or at least that Amazon vegetation will become unsustainable [4]. Modelers have recognized that to increase the precision of model outputs it is necessary to refine the physiological response of vegetation to climatic variability [5]. Dynamic Global Vegetation Models (DGVMs) which represent recycling of greenhouse gases between soils and the atmosphere, usually simulate the characteristics of the annual vegetation phenology cycle to represent key events such as the onset of greenness. The accuracy of the phenology simulation depends on the understanding the dominance of biophysical variables controlling phenology, and understanding this on a global scale is a challenge. Satellite data can be used to measure phenology patterns and these data have potential to assist the validation and parameterization of DGVMs. Many researchers have monitored and characterized phenology with remote sensing data, e.g. [6] [7], using vegetation indices such as NDVI and EVI. These time series (figure 1 and 2) can be compared to other satellite derived time series such as precipitation from the Tropical Rainforest Monitoring Mission (TRMM) [8], and radiation from the Clouds and the Earth's Radiant Energy System mission (CERES) [9]. With this synoptic view we can begin to understand the relations between phenology and their drivers in a spatial and temporal context. Our objective is to compare a time series of phenology against two biophysical variables, precipitation (Ppt) and net radiation $(\mathrm{Rn})$, and understand the relationships and spatial patterns between, (A) the strength of the annual cycles to give an indication of where and how influential the biophysical driver is and, (B) how closely the annual cycles are in amplitude (coherency) and timing (phase). Where these time series have good coherency there is potential to show cause and effect relations with the offset in the timing between the two time series (phase). We predict that if a biophysical driver controls the phenology the strength of the annual phenology cycle has a positive correlation with the strength of the biophysical driver and that if a biophysical driver has a cause effect relationship with phenology the biophysical variable will slightly precede the phenology cycle. We test these ideas by passing a Fourier transform over the time series and then apply a cross spectral analysis [10]. We examine our results spatially and indicate where and how the two biophysical variables have influenced phenology with the results we have produced so far.

\section{STUDY AREA}

The study region is focused on the South America tropics, north west corner: $10.0^{\circ} \mathrm{N}, 81.0^{\circ} \mathrm{W}$ and south east corner: $20.0^{\circ} \mathrm{S}$, $40.0^{\circ} \mathrm{W}$. Models have predicted a severe dieback in this area by 2050 so it is logical that we assist any validation / model parameterization in such a critical area.

\section{DATA}

For the phenology we downloaded $1 \mathrm{~km}$ by $1 \mathrm{~km}$ NDVI and EVI 16 day MODIS composites from the NASA LPDAAC archive giving 158 time points between the dates April 2000 to Dec 2007. 


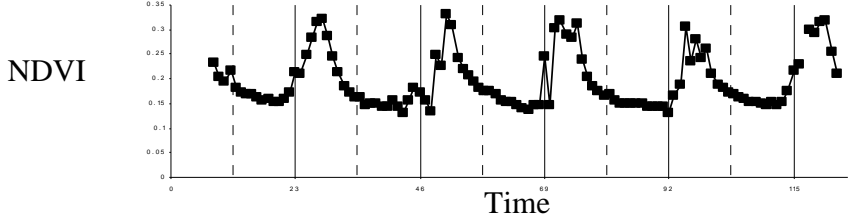

Figure 1. Typical highland grassland phenology cycle

NDVI

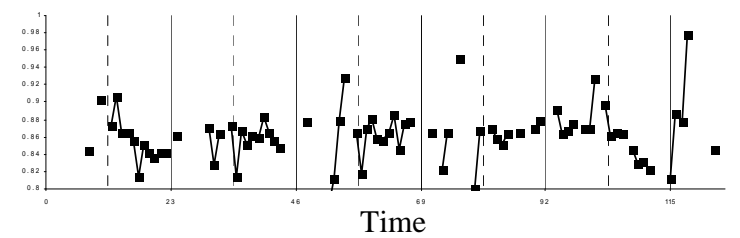

Figure 2. Typical tropical moist forest phenology cycle

These time series were cleaned to remove data gaps and outliers by averaging the bimonthly composites into monthly composites. For monthly precipitation (Ppt) data we used the TRMM data and other sources rainfall data set at $0.25^{\circ}$ by $0.25^{\circ}$ resolution acquired from the Goddard Distributed Active Archive Center for the corresponding time period to the vegetation indices. To calculate the net radiation $(\mathrm{Rn})$ budget we used data at $1^{\circ}$ by $1^{\circ}$ resolution from the CERES mission using the FSW (monthly gridded radiative fluxes and clouds) product. These data were obtained from the NASA Langley Research Center Atmospheric Science Data Center. Daily average Rn was calculated for a full year, 2002, using a sinusoidal model [11] and to avoid the bias of day length radiation for each grid cell was calculated to a 24 hour average and then monthly averages were calculated. To match the length of the phenology time series the 2002 data was replicated and stacked, in the future this will be replaced with all 4.8 years of data from March 2000 (missing June 2001). For time series analysis of radiation against phenology we used a readily available radiation data set of average monthly total radiation, CIPEC, acquired from the INPE (National Institute for Space Research, Brazil). Although the resolution $0.4^{\circ}$ by $0.4^{\circ}$ is higher than the FSW data the CIPEC archive is restricted to a smaller area corresponding to the north east of the main study area.

\section{METHOD}

This method was designed to compare: (A) the presence, strength and location of the annual cycle and; (B) coherency and phase differences between the phenology data and the biophysical drivers, Ppt and Rn. For Part A each time series was de trended and tapered before the Fourier transform was passed over the time series [10] then to synchronize the annual power output over the study area we passed a peak find algorithm on the $7^{\text {th }}$ period of the periodogram (equivalent to an annual cycle). Pixels with no annual peak were discarded. The results were then re sampled to the lowest resolution dataset and mapped to show the spatial variation in annual power for each data set. These data were divided with cumulative breaks of $33 \%$ and $66 \%$ and classified into intervals of low, medium and high power, then multiplied together and mapped to show the correspondence in annual power between the phenology and each biophysical driver. In Part B for each data set we applied a Fourier transform to calculate the power spectra of each time series. Then, after resampling to the lowest resolution dataset, using a cross spectral analysis we compared the phenology periodogram against Ppt then Rn periodograms to yield coherency and phase values. These data were then mapped to identify spatial differences in similarity and timing of the phenology against the biophysical drivers.

\section{RESULTS}

\subsection{Part A: location and relative strength of annual cycles}

The distribution of identified annual peaks and their power for phenology and Rn are shown in figures 3 and 4. In the phenology (figure 3 ) areas with high power $>\sim 0.18$ show where the annual cycle is pronounced: a patch in the northwest; most of the south east and; a line trending NW to SE in the south west of the study area. These locations correspond to areas of shrub, savanna and high altitude grasslands in the Andes, e.g. figure 1. Areas with low power $<\sim 0.06$ indicate where the annual cycle is subdued: this characteristic dominates the central west of the study region, and these areas correspond to the tropical moist forest e.g. figure 2. Areas with an intermediate power range characteristic of slightly subdued annual cycles $\sim 0.06$ to $\sim 0.18$ graduate between the high and low power areas and tend to dominate the central east. These areas tend to reflect the transition from tropical moist forest through dry forest and shrub to savanna and include deforested areas now replaced with agriculture. In the Rn (figure 4) pronounced annual cycles, power $>\sim 0.21$ are found in: an area running from the Atlantic Ocean through the central north and central north west; in the far central south running up the Andes and into the Pacific Ocean, on land these areas cross the transition of dry and moist forests and the montane grasslands and deserts respectively. Areas of low power $<\sim 0.04$, with a subdued annual cycle occur in: a large area slightly south east of the centre which crosses moist forest and shrub land and; a narrow north to south belt on the eastern margins of the Andes where there is mainly montane and moist forest. The areas of intermediate power and slightly subdued annual cycles, between $\sim 0.04$ and $\sim 0.21$, tend to join the two low power areas or form a gradient between the high and low power areas. The correspondence in the strength of phenology and $\mathrm{Rn}$ power is shown in figure 5. There are areas where both data sets have good correlation of high power, light red $(\mathrm{H} \vee \mathrm{H})$, and low power, dark purple ( $\mathrm{L} \vee \mathrm{L}$ ), conversely there are areas where power strengths are weakly linked, dark red $(\mathrm{H} v \mathrm{~L})$ and light purple ( $\mathrm{L} v \mathrm{H}$ ). This figure shows that the strength of the annual phenology cycle is not always linear with the strength of the $\mathrm{Rn}$ cycle indicating other factors are suppressing this relationship. It is expected that the analysis of the strength of annual Ppt may in part explain this.

\subsection{Part B: coherence and phase differences}

Figure 6 shows a map of the coherency between EVI and Ppt (TRMM) data for the annual cycle. The values range from 0 , no coherency, to 1, perfect coherency. Patterns were similar for the NDVI-Ppt result in that the core areas of low coherency were the same as the EVI-Ppt result, but their extent was smaller indicating that EVI is more sensitive to the comparison. For this reason and to maintain a concise review we will report the EVI results. The areas of high coherency tend to be in the northwest central, eastern and south eastern areas. The main areas of low coherency were in the central west, and a core in the north central area. 


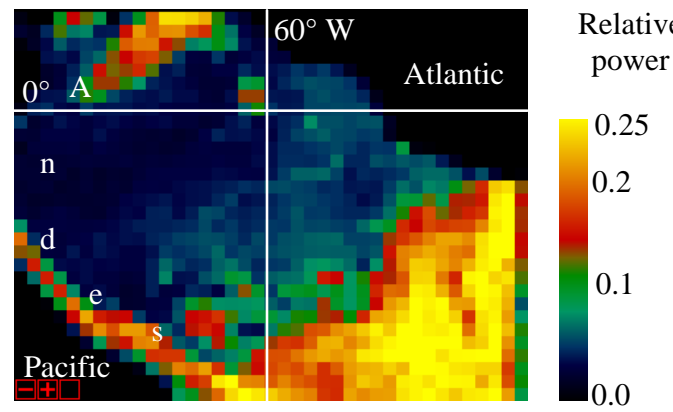

Figure 3. Phenology annual power $\left(1^{\circ}\right.$ resolution $)$

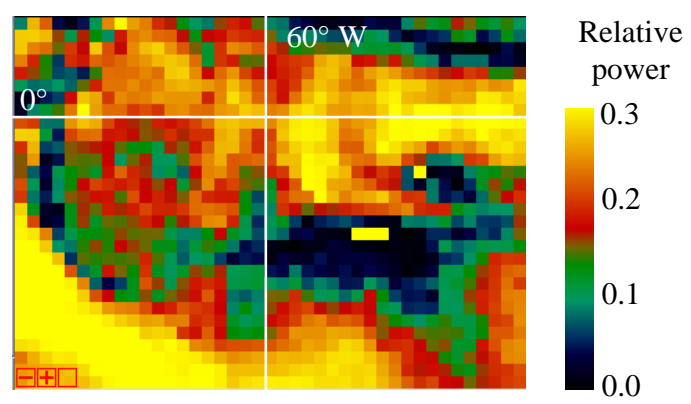

Figure 4. Rn annual power $\left(1^{\circ}\right.$ resolution)

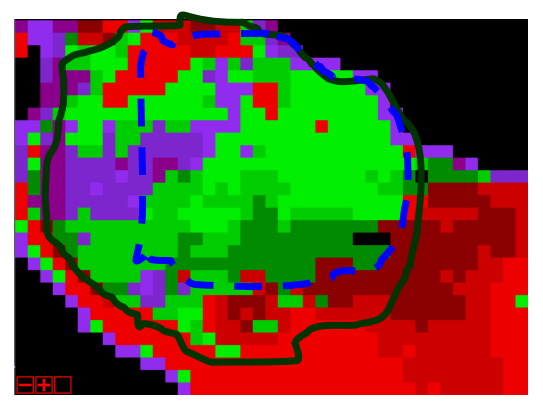

Relative power

matrix

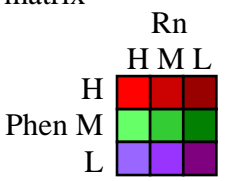

Figure 5. Correspondence between phenology and Rn annual power ( $1^{\circ}$ resolution). Matrix: $\mathrm{H}$ - high, $\mathrm{M}$ - medium, $\mathrm{L}-$ low. Outlines: solid figure 7; stippled figure 8

In the areas where there is a high coherency (where the time series periodograms have a similarity in amplitude) we can be confident about the phase shift between the annual peaks and examine the cause and effect relations of Ppt on phenology conversely in low coherency areas the dominance of Ppt on phenology weakens and other cause and effect relations should be explored. We focus on the area of high coherency to examine the phase shifts (timing) between the two time series. A map of these phase differences shows how the phase differences vary spatially (figure 7). To the north of the Amazon river (east west trending linear feature masked from the analysis) the darker blue areas show where phenology precedes Ppt by up to six months $(\sim+3.140943$ radians $=+6$ months) and the darker brown area running east to west across the centre of the area show where Ppt precedes the phenology by up to four months ( $\sim 2.09$ radians $=-4$ months). Towards the south and west of the image the phase difference tends closer to zero, which extends to the Andes and central north (not shown). This indicates that the cause and effect between Ppt and phenology is stronger to the fringes of the Amazon but quite weak in the central and eastern areas, indicating other biophysical

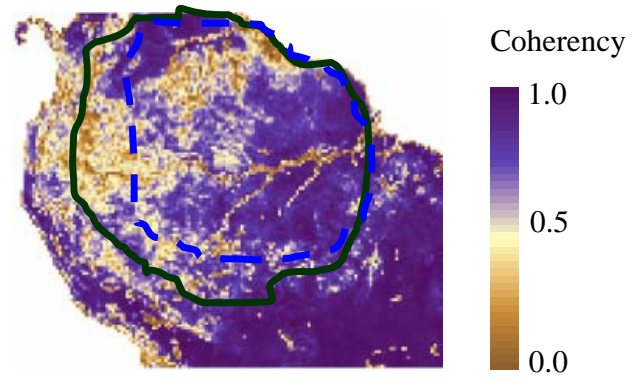

Figure 6. Coherency between EVI and Ppt (0.25 resolution)

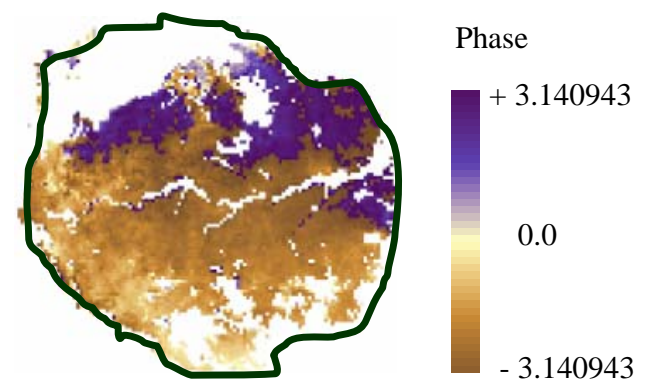

Figure 7. Phase difference between EVI and Ppt (0.25 resolution)

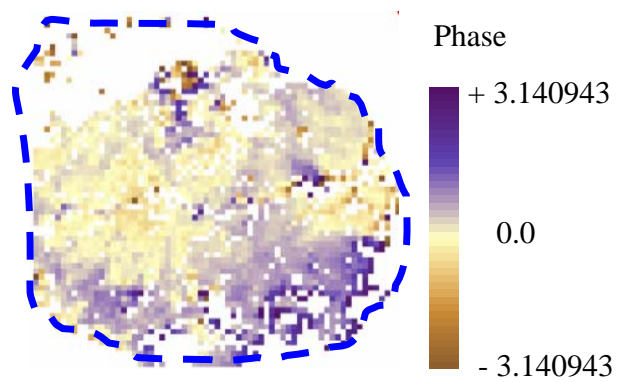

Figure 8. Phase difference between EVI and Rn $\left(0.4^{\circ}\right.$ resolution)

drivers are in the frame. Figure (8) shows the phase differences between EVI and Rn. Here the analysis is constrained to the bounds of the CIPEC data (see method) and is slightly less that figure 7. The map (figure 8) indicates that EVI and Rn are almost in phase with EVI slightly preceding $\mathrm{Rn}$ by less than half a month ( 0.25 radians) and this relationship dominates throughout the central parts of the study location but weakens in the central east, to the south and south east. Comparing phase shifts with Ppt (figure 7) and Rn (figure 8) we see that where there is little phase difference between $\mathrm{Rn}$ and EVI, there is a phase difference between Ppt and EVI and where the phase difference between EVI and $\mathrm{Rn}$ weakens (south and south east), the phase difference between EVI and Ppt begins to tend towards zero. In this particular area these results indicate that $\mathrm{Rn}$ is a major phenology driver in central parts whilst Ppt becomes more dominant to the south (figure 8) and at the fringes of the whole study area (not shown).

\section{DISCUSSION}

Revisiting our original hypothesis the current progress of this research has shown us that the strength of the annual phenology 
cycle does not always correspond to the strength of the annual cycle of a biophysical driver. Where a driver has a strong cycle it does not always force a strong phenology cycle and conversely where the driver is weak strong phenology cycles can exist showing that the driver may have a weaker or more dominant effect in different areas. The results presented here are against one driver, Rn, we are now investigating another driver, Ppt and it remains intriguing to see if the cross correlation in the annual power of TRMM data will replicate or show quite different patterns from figure 5. Whatever the outcome it will show where in the Amazon the annual signals in biophysical drivers are most pronounced, and of even more interest if all or some biophysical drivers are pronounced or subdued in the same or different locations. The coherency and phase results show in some areas there is a strong cause and effect relationship between the driver and phenology, and this identifies locations where a driver is dominant over other drivers. In our intensively studied area we have shown spatially how Rn can be in phase and Ppt can be out of phase with phenology in the same locations. However being in phase does not necessarily mean a pronounced annual phenology peak at that location. Our current explanations are of course limited to the drivers that we have analyzed and can analyze with satellite data, so although Rn and phenology were almost in phase there will be other mechanisms influencing the strength of the phenology cycle, e.g. soils, topography, and land use. Soils would have an effect on the soil moisture deficit, which could be explored with continental scale FAO soil maps. In some areas agriculture may dominate and phenology may be more closely related to cropping patterns and we expect a poor correspondence with our 'natural' biophysical drivers. There is also the possibility, particularly as this is an equatorial region that a phenology cycle with a different periodicity, perhaps biannual, may exist in some areas and will drive the phenology strength. Further comparison and cross mapping of phase differences in other drivers will spatially show where different drivers can dominate, or contribute to the phenology cycles.

\section{CONCLUSION}

Using a time series of MODIS data, CERES net radiation data and TRMM precipitation data in the region of the Amazon, we have identified using a Fourier transform (i) the presence and spatial distribution in the power of the annual cycle of phenology and net radiation and, (ii) coupled with a cross spectral analysis, identified coherency in areas where phenology and a biophysical driver are closely matched. Phase differences have shown us patterns of cause and effect between the biophysical and phenology. We have shown that where there is a strong coherency between phenology and net radiation and phenology and precipitation, phase differences can vary spatially. We have also shown that a strong annual signal which is in phase with phenology does not necessarily cause a strong annual phenology signal, indicating the partial contribution of specific biophysical drivers to phenology. Continued exploration of the biophysical variables will provide modelers with a better understanding of the drivers of phenology.

\section{ACKNOWLEDGEMENTS}

This research has been supported by the Natural Environment Research Council (NERC), Centre for Ecology and Hydrology, a
NERC funded project QUEST QUERCC [5] and the Environmental Change Institute, Oxford, UK.

\section{REFERENCES}

[1] Solomon, S., Qin, D., Manning, M., Marquis, M., Avery, K., Tignor, M.M.B., LeRoy Miller, H. and Chen, Z. (eds), "Climate Change 2007: The Physical Basis", Cambridge University Press, Cambridge, UK, 996pp. 2007

[2] Cox P.M., Betts R.A., Jones C.D., Spall S.A. \& Totterdell I.J., "Acceleration of global warming due to carbon cycle feedbacks in a coupled climate model.” Nature, 408, 184-187, 2000

[3] Murphy, J.M., Booth, B.B.B., Collins, M., Harris, G., Sexton, D. and Webb, M., "A methodology for probabilistic predictions of regional climate change from perturbed physics ensembles". Philosophical Transactions of the Royal Society A 365: 1993-2028. 2007

[4] Salazar, L.F., Nobre, C.A. and Oyama, M.D., "Climate change consequences on the biome distribution in tropical South America”, Geophysical Research Letters, 34, doi: 10.1029/2007GL029695, 2007

[5] Quantifying Earth system processes and feedbacks for better informed assessments of alternative futures of the global environment (QUEST), http: // quest.bris.ac.uk /index.html, Quantifying ecosystem roles in the carbon cycle (QUERCC), http : // quest.bris.ac.uk /research /themes / QUERCC .html, Department of Earth Sciences, University of Bristol, Wills Memorial Building, Queen's Road, Bristol, BS8 1RJ

[6] Heumann, B. W., J. W. Seaquist, et al., "AVHRR derived phenological change in the Sahel and Soudan, Africa, 1982-2005." Remote Sensing of Environment 108 (4): 385-392. 2007

[7] Geerken, R., B. Zaitchik, et al., "Classifying rangeland vegetation type and coverage from NDVI time series using Fourier Filtered Cycle Similarity." International Journal of Remote Sensing 26 (24): 5535-5554. 2005

[8] Kummerow, C. , W. Barnes, T. Kozu, J. Shiue, and J. Simpson., "The Tropical Rainfall Measuring Mission (TRMM) Sensor Package" Journal of Atmospheric and Ocean Technology., 15, 808-816. 1998

[9] Wielicki, B. A., B. R. Barkstrom, E. F. Harrison, R. B. Lee III, G. L. Smith, and J. E. Cooper., "Clouds and the Earth's Radiant Energy System (CERES): An Earth Observing System Experiment," Bulletin American Meteorological Society, 77, 853868, 1996

[10] Weedon, G., "Time-Series Analysis and Cyclostratigraphy”. Cambridge University Press, pp 259. 2003

[11] Bisht, G., Venturini, V., Islam, S., Jiang, L., "Estimation of the net radiation using MODIS (Moderate Resolution Imaging Spectroradiometer) data for clear sky days”, Remote Sensing of Environment 97, 52-67, 2005 